\title{
A integralidade nas ações da equipe de saúde de uma unidade de internação pediátrica
}

\author{
Débora Luíza dos Santos ${ }^{1}$ \\ José Luís Guedes dos Santos ${ }^{2}$ \\ Adelina Giacomelli Prochnow ${ }^{3}$ \\ Maria de Lourdes Rodrigues Pedroso ${ }^{4}$ \\ Maria Alice Dias da Silva Lima ${ }^{5}$
}

SANTOS, D.L. et al. Comprehensiveness of the actions of the healthcare team in a pediatrics ward. Interface - Comunic., Saude, Educ., v.13, n.31, p.359-68, out./dez. 2009.

The notion of comprehensiveness encompasses three sets of meanings: the practices of healthcare professionals, the attributes of the service organization and the government responses to health-related problems. This study had the aim of analyzing and discussing the meanings relating to comprehensiveness of the actions of the healthcare team in a pediatrics ward of a university hospital in the interior of the state of Rio Grande do Sul. This was a qualitative study in which data were collected through interviews and analyzed through thematic analysis. The comprehensiveness of professionals' practices is focused on the dimension relating to professional action, in which they seek to associate assistance and prevention, considering the hospitalized children within their social and economic contexts. The importance of team work is highlighted, but difficulties in making this effective were found. These difficulties impair the interlinking of actions towards comprehensive healthcare.

Keywords: Comprehensive healthcare. Patient caret. Professional practice. Hospital services. Pediatrics.

\begin{abstract}
A noção de integralidade engloba três conjuntos de sentidos: a prática dos profissionais de saúde, os atributos da organização do serviço e as respostas governamentais aos problemas de saúde. Este estudo teve como objetivo analisar e discutir os sentidos relacionados à integralidade nas ações da equipe de saúde de uma unidade de internação pediátrica de um hospital universitário no interior do estado do Rio Grande do Sul. Trata-se de uma pesquisa qualitativa cujos dados foram coletados a partir de entrevistas e analisados mediante análise temática. A integralidade na prática dos profissionais está centrada na dimensão relativa à atuação profissional, em que eles buscam associar assistência e prevenção, vislumbrando a criança hospitalizada no seu contexto econômico e social. Apontam a importância do trabalho em equipe, mas encontram dificuldades em efetivá-lo, o que prejudica a articulação das ações em prol de uma atenção integral.

Palavras-chave: Assistência integral à saúde. Equipe de assistência ao paciente. Prática profissional. Serviços hospitalares. Pediatria.

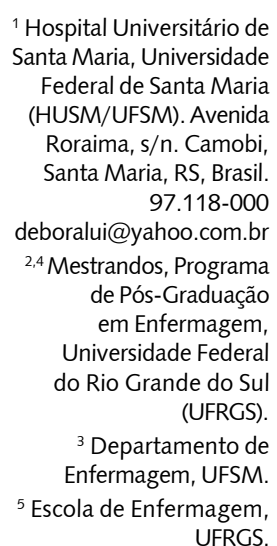




\section{Introdução}

A Constituição Federal, promulgada em 1988, define saúde como um dever do Estado e um direito da população, e estabelece que as ações e os serviços de saúde têm importância pública e devem ser promovidos por um Sistema Único de Saúde (SUS), organizado em torno dos princípios da universalidade, integralidade e equidade. No entanto, a promulgação desses direitos constitucionais e as políticas públicas de saúde não impactaram mudanças efetivas no modo de produzir saúde, de tal forma que um dos principais desafios no cotidiano atual dos serviços de saúde é a produção de práticas de saúde segundo os preceitos do SUS (Pinho, Siqueira, Pinho, 2006).

Muitos esforços têm sido empregados na busca da reestruturação dos serviços e das ações em saúde por meio de políticas públicas, de forma a fazer com que os profissionais atuem sob uma lógica de atenção voltada para a integralidade da assistência. No entanto, a modificação da estrutura e das formas de organização dos serviços não assegura mudanças nos modelos assistenciais e suas micropolíticas instituídas, pois os comportamentos e valores dos profissionais de saúde influenciam o modo como se processam essas modificações (Franco, Merhy, 2004).

Logo, estratégias que busquem viabilizar arranjos diferenciados para os serviços de saúde são necessárias para a construção de uma lógica diferenciada de atenção, assim como a discussão do trabalho em saúde voltado para a integralidade da assistência e responsabilização dos profissionais com a produção de saúde que valorize e oportunize maior autonomia ao usuário.

Nessa ótica, cabe ressaltar que as características de determinado processo de trabalho são, muitas vezes, determinadas pelo autogoverno do trabalhador de saúde sobre o modo de fazer a assistência. Durante a realização das suas atividades, ele pode agir como um dispositivo gerador de mudanças e modificação dos processos ante a organização dos serviços de saúde, tendo em vista que a realização do trabalho vivo em ato pressupõe a possibilidade da criatividade, a qual pode ser utilizada para inventar novos processos de trabalho (Marques, Lima, 2008; Merhy, 1997). Portanto, a organização do processo de trabalho em saúde tanto contribui para a manutenção do modelo hegemônico, como constitui um campo profícuo para a construção de dispositivos de mudança com relação à lógica instituída na produção de cuidados de saúde.

Tal fato baliza a importância da compreensão dos significados relacionados à integralidade no trabalho em saúde para os profissionais que o executam, no intuito de suscitar a discussão acerca dos mecanismos necessários à construção e efetivação das práticas de integralidade no interior dos serviços de saúde em seus diferentes níveis de complexidade.

A noção de integralidade nos serviços de saúde engloba três conjuntos de sentidos distintos, mas complementares: a prática dos profissionais de saúde, os atributos da organização do serviço e as respostas governamentais aos problemas de saúde. O primeiro sentido está relacionado à postura do profissional, a qual deve buscar apreender o contexto de vida dos pacientes, considerando-os muito mais que lesões ou disfunções a serem tratadas, para isso é necessário associar assistência com prevenção. No que tange à organização dos serviços, aponta-se que o modo de organizar deve ser aberto a assimilar as necessidades dos usuários e pautado no diálogo entre os diferentes sujeitos que compõem o processo de trabalho em saúde. Quanto às respostas governamentais, elas devem considerar as especificidades dos grupos atingidos pelos problemas de saúde, a partir da contextualização dos sujeitos sobre os quais as políticas incidem (Mattos, 2004, 2001).

Partindo da dimensão das práticas de integralidade enquanto norteadoras das ações dos trabalhadores em saúde, compreende-se o acolhimento, a desfragmentação do atendimento prestado e a integralidade como um processo de construção social, permeado de uma maior horizontalidade nas relações entre os seus participantes, incorporando continuamente novos conhecimentos a partir das práticas dos sujeitos envolvidos na produção do cuidado em saúde (Pinheiro, Mattos, 2006).

No entanto, na saúde em geral e, mais especificamente, na saúde da criança, são muitos os obstáculos a serem enfrentados para que sejam implementadas ações com vistas a um novo paradigma que não aquele que prioriza somente a doença, introduzindo conceitos e ações facilitadores de promoção da saúde, como a integralidade. 
$\mathrm{Na}$ atenção à saúde da criança, há estreita relação entre a educação e a promoção da saúde, visto que as ações implementadas em todos os níveis de atenção, além de tratar e/ou prevenir agravos, destinam-se, também, a promover o crescimento e desenvolvimento infantil, numa perspectiva de qualidade de vida. As ações de promoção da saúde devem ser acionadas por meio de estratégias que envolvam a coletividade em geral e a família, a qual é responsável pela criança e possuidora de um saber que não deve ser descartado, mas aperfeiçoado e/ou adaptado ao saber científico dos profissionais (Queiroz, Jorge, 2006).

Além disso, existem algumas particularidades do atendimento infantil que não contemplam o princípio da integralidade, dentre as quais se destaca o papel reservado às crianças nos serviços de saúde. Mesmo quando elas têm condições de opinar sobre seu estado de saúde e participar de decisões sobre seu tratamento, suas manifestações são, constantemente, mediadas pelos adultos responsáveis ou checadas com eles. Médicos, por exemplo, solicitam às crianças que descrevam os seus sintomas, mas tendem a excluí-las das informações referentes ao diagnóstico e à terapêutica, restringindo sua interação ao afetivo/lúdico no intuito de agradá-las. Entretanto, uma maior participação da criança é fundamental para a satisfação e adesão ao tratamento, possibilitando um melhor prognóstico (Perosa, Ranzani, 2008; Perosa, Gabarra, 2004).

Ressalta-se que a integralidade é um dos princípios que norteia não apenas as práticas realizadas nas unidades de saúde, mas toda a produção do cuidado nas instâncias do SUS. Assim, justifica-se a relevância de se investigar a integralidade com base na visão de profissionais de saúde que atuam no contexto hospitalar, tendo em vista que a maioria dos estudos relacionados a essa temática têm sido realizados no âmbito da atenção primária.

Desse modo, este estudo teve como objetivo analisar e discutir os sentidos relacionados à integralidade nas ações dos profissionais da equipe de saúde de uma unidade de internação pediátrica de um hospital universitário.

\section{Metodologia}

Trata-se de um estudo exploratório-descritivo com abordagem qualitativa, que buscou entender a relação entre o trabalho de profissionais de saúde no contexto hospitalar e os sentidos da integralidade presentes em suas ações. O método qualitativo permite estudar os significados que as pessoas atribuem a um dado fenômeno, em torno dos quais elas organizam de certa forma suas vidas e atitudes em relação à saúde (Turato, 2003).

O cenário de estudo foi uma unidade de internação pediátrica de um hospital universitário localizado no interior do estado do Rio Grande do Sul (RS), o qual integra a rede pública de saúde enquanto hospital de referência em nível terciário para toda a região centro-oeste do estado. Esse cenário foi escolhido para o desenvolvimento da pesquisa tendo em vista a importância da integralidade nas ações dos profissionais de saúde na atenção às especificidades que envolvem o cuidado à criança hospitalizada, como: o atendimento de suas necessidades sociais, intelectuais, emocionais e recreacionais durante a internação, bem como a atenção dispensada aos pais e familiares durante esse processo.

Os sujeitos da pesquisa foram selecionados de forma intencional, tendo em vista as atividades laborais que desenvolviam no cenário do estudo. Assim, participaram da investigação seis trabalhadores de saúde de nível superior, sendo: dois enfermeiros, dois médicos, psicólogo e nutricionista. A amostra foi constituída de forma a compor um universo multiprofissional, objetivando a identificação dos significados da integralidade nas ações em saúde sob diferentes olhares dentro de um mesmo universo de práticas, sem predominância de uma categoria profissional em relação à outra. Salienta-se que os profissionais fisioterapeutas não participaram do estudo em virtude da incompatibilidade de horários entre esses trabalhadores e os pesquisadores no período em que a pesquisa foi realizada.

Para a coleta de dados, optou-se pela técnica da entrevista semiestruturada, na qual os participantes do estudo foram convidados a responder sete questões, as quais exploravam as suas percepções quanto 
à relação saúde e doença, o processo de trabalho e os sentidos da integralidade nas práticas que eles desenvolviam no seu cotidiano laboral em pediatria. Salienta-se que, no intuito de garantir maior autonomia e anonimato aos entrevistados, as entrevistas foram realizadas por dois acadêmicos de enfermagem, os quais não possuíam vínculo com a unidade e os profissionais. As entrevistas foram gravadas e transcritas posteriormente, sendo identificadas por meio de códigos compostos pela sigla "Prof.", referente a profissional, associada a letras do alfabeto atribuídas segundo a ordem na qual os depoimentos foram coletados (Prof. A, Prof. B, ...).

Para analisar os dados, fez-se uso da análise temática, que se constitui de três etapas: ordenação, classificação dos dados e análise final (Minayo, 2007). Os dados foram organizados e discutidos de acordo com os três conjuntos de sentidos da integralidade propostos por Mattos $(2004,2001)$.

Com relação às questões éticas, ressalta-se que o presente estudo foi aprovado pela Direção de Ensino, Pesquisa e Extensão do hospital em que a pesquisa foi realizada e pelo Comitê de Ética da universidade à qual essa instituição está vinculada academicamente (Parecer $n^{\circ}$ 0163.0.243.000.06). Além disso, foi solicitada a autorização dos participantes do estudo para sua inclusão na pesquisa, mediante apresentação do Termo de Consentimento Livre e Esclarecido de acordo com a Resolução 196/96 do Conselho Nacional de Saúde (Brasil, 1996).

\section{Apresentação e discussão dos resultados}

Para analisar os significados da integralidade, enquanto princípio das ações em saúde, primeiro partiu-se da identificação do conceito de saúde como produto das ações desenvolvidas pelos profissionais de saúde entrevistados, e do significado do termo integralidade no universo das práticas desenvolvidas no cenário investigado.

Considerando-se a noção de integralidade pautada em três conjuntos de sentidos distintos, com base na análise e interpretação das ações relatadas pelos profissionais evidenciou-se que o conceito de integralidade é mais facilmente associado à dimensão relativa à atuação profissional da equipe de saúde.

Os entrevistados definiram saúde de forma ampla, como sendo não apenas a ausência de doença, mas uma relação equilibrada entre diversos fatores - como alimentação, atividade física, condições adequadas de saneamento básico, assim como aspectos mentais e sociais - os quais, em conjunto, são capazes de proporcionar qualidade de vida, bem-estar e, consequentemente, saúde ao indivíduo:

"É o bem-estar físico, social, cultural da pessoa, o mais abrangente possível." (Prof. A)

\footnotetext{
"Bem-estar conjunto, não é apenas ausência de patologia, envolve também o ambiente em que a pessoa vive." (Prof. B)
}

Esses conceitos apresentados vão ao encontro da definição de saúde expressa pela Organização Mundial de Saúde e sustentam a problematização das ações segundo uma abordagem pautada na integralidade.

Com relação às finalidades do trabalho, os profissionais relataram o desenvolvimento de ações que vão além das atividades de recuperação no âmbito hospitalar, apontando para a amplitude das necessidades de saúde dos usuários, o que demonstra que a noção de integralidade está presente nas práticas que eles desenvolvem:

\footnotetext{
"Procurar o bem-estar, qualidade de vida, procurar não contribuir apenas no hospital." (Prof. C)

"[...] proporcionar conforto ao paciente que está internado na unidade, tentar que ele saia daqui totalmente recuperado e orientar os pais quanto aos cuidados após a alta também." (Prof. D)
} 
“Procurar promover saúde, buscar os aspectos que envolvem a saúde, o bem estar não só físico, mas em todos os aspectos." (Prof. F)

Os profissionais tanto referiram, na base conceitual de suas práticas como nas suas finalidades, a integralidade enquanto fundamento norteador, a partir da articulação de ações de assistência e prevenção. De forma semelhante, enfermeiras de unidades de saúde de Goiânia entrevistadas em relação às suas percepções quanto à integralidade apontaram que a prática integral consiste no atendimento globalizado e holístico dos pacientes (Pinho, Siqueira, Pinho, 2006).

Quanto às práticas nas quais conceitos e objetivos de integralidade são efetivados, encontrou-se a procura por ações que extrapolem o contexto hospitalar e busquem situar o usuário no seu contexto social mais amplo:

“[...] Quando avaliamos o paciente na pediatria, queremos saber onde é que ele mora, como é que é a casa, quais pessoas que moram na casa, a vida social desse paciente, alimentação, enfim, tudo. Na pediatria, nós olhamos todo o paciente, e na medida do possível tentamos melhorar essas deficiências, até encaminhar ele para o serviço que ele precisa." (Prof. E)

"Inclui esta inter-relação, esta troca permanente, questionamentos com outros profissionais sobre crianças negligenciadas, problemas sociais, não basta apenas tratar a doença, sem investigar o que a determinou." (Prof. F)

A contextualização social da criança, entendida como a investigação do ambiente familiar e estrutural em que vive, foi apontada como uma estratégia fundamental à continuidade do tratamento prescrito e orientado no contexto hospitalar. De acordo com os entrevistados, a partir dela, podem-se captar as necessidades dos pacientes que nem sempre estão explícitas, interferindo sobre elas ou realizando os encaminhamentos necessários.

Nesse sentido, salienta-se a necessidade do estabelecimento de projetos terapêuticos baseados no diálogo entre trabalhadores de saúde e usuários, no qual os profissionais devem se esforçar no sentido de buscar os elementos centrais de cada encontro, tanto com base no seu conhecimento como nos saberes trazidos pelo usuário, e pelo seu contexto específico (Mattos, 2004).

Seguindo essa linha reflexiva, com relação à comunicação entre os profissionais de saúde e os usuários do serviço de internação infantil em que o estudo foi realizado, salienta-se que, na falas dos depoentes, predominam menções à busca de informações com os familiares, de maneira que o processo interativo com a criança hospitalizada parece estar em segundo plano. Entretanto, a hospitalização representa uma situação estressante e, muitas vezes, geradora de sofrimento e medo às crianças, o que as faz desejar informações acerca: do seu estado de saúde, dos procedimentos diagnósticos aos quais são submetidas, de quem são os profissionais que cuidam delas, e de qual efeito terão as ações terapêuticas desempenhadas em prol da sua recuperação (Ribeiro, Angelo, 2005; Soares, Vieira, 2004).

Analisando o enfoque do trabalho em equipe nas práticas de integralidade, questionaram-se os profissionais quanto à forma como eles atuavam cotidianamente. Nas respostas, eles revelaram dificuldade de interação na unidade, apontando, como obstáculos, a ausência de construções coletivas diárias e as barreiras de comunicação entre os diferentes profissionais, como, por exemplo, médicos e enfermeiros no que tange à realização de procedimentos e transferência ou alta dos pacientes.

Além disso, evidenciou-se que as ações descritas por eles como trabalho em equipe, ao serem analisadas, configuraram-se como um trabalho coletivo, realizado a partir da união de práticas isoladas:

"A psicóloga, às vezes, chama a atenção para aspectos que são importantes para aquela criança, como a perda dos pais, maus tratos, pois os médicos nem sempre têm tais informações." (Prof. D) 
"Aqui a gente tem apoio bastante da nutrição e da psicologia, a gente pede e elas vão de imediato já avaliar e ajudar. E da enfermagem também, a gente tem bastante contato, e a secretária também, que ajuda e muito." (Prof. E)

Reforça-se, portanto, a percepção de que os profissionais buscam, de forma isolada, uma assistência integral à criança hospitalizada. Cada um desenvolve suas ações no seu campo de atuação, com o apoio dos demais profissionais em suas respectivas áreas sem, entretanto, efetivar um trabalho de discussão, planejamento e execução de atividades de forma articulada e pautadas na interdisciplinaridade.

Entretanto, Menossi, Lima e Correia (2008) asseveram que a prática interdisciplinar com base na articulação dos diversos saberes, em torno de um projeto comum, é essencial para que sejam contempladas as múltiplas dimensões que envolvem o cuidado à criança e ao adolescente hospitalizado. Nesse sentido, o trabalho realizado em equipe, de forma articulada, pautado na interação entre os profissionais, pode favorecer a atenção integral à saúde da criança.

O trabalho em equipe refere-se à relação entre trabalho e interação de agentes técnicos distintos, mas sujeitos iguais. Por interação entende-se a construção de consensos quanto aos objetivos e resultados a serem alcançados pelo conjunto dos profissionais. A partir dessa prática comunicativa, os profissionais elaboram e executam um projeto comum que contempla as necessidades de saúde dos usuários (Peduzzi, 2001).

O trabalho integrado só foi evidenciado em momentos típicos de emergência, no qual apresenta-se certa quebra da dificuldade de interação dos profissionais, em que a equipe atua de forma solidária, como pode ser constatado na seguinte fala:

\footnotetext{
"Nós estávamos com uma criança ruim e daí toda equipe parou: era um chamando médico, um administrando medicação, um verificando sinais, todos agiram junto como uma equipe mesmo." (Prof. B)
}

A situação acima foi descrita pelo profissional com o intuito de exemplificar o trabalho em equipe destinado ao atendimento integral a uma criança durante o atendimento a uma emergência clínica. No entanto, destaca-se que a interação à qual a fala remete está centrada na realização de procedimentos técnicos de forma articulada. Sabe-se que, durante o atendimento a uma emergência, a aferição dos sinais vitais e a administração de medicamentos são extremamente importantes; no entanto, trabalhar em equipe pressupõe não só a realização conjunta de atividades técnicas, mas também, e sobretudo, a discussão dos problemas e das necessidades da equipe e dos pacientes atendidos na busca de consensos coletivos.

Nesse sentido, constata-se que o processo de trabalho, no contexto hospitalar, está pautado basicamente em atividades assistenciais, diferenciadas de acordo com cada grupo profissional, as quais, do ponto de vista do cuidado à saúde, articulam-se em uma rotina de trabalho coletivo, constituindo-se em ações fragmentadas, realizadas de maneira conjunta (Martins, 2004).

Apesar do exposto, quando questionados acerca dos elementos capazes de tornar mais eficaz e integral o seu trabalho, os profissionais salientaram a importância da interdisciplinaridade e atuação em equipe, assim como da comunicação no ambiente de trabalho:

“Integração entre profissionais de diferentes áreas, comunicação, colaboração destes profissionais, instrumentos de trabalho e um bom relacionamento com a equipe e paciente." (Prof. C)

“[...] comunicação entre a equipe, 'mensagem uniforme' ou seja, todos os profissionais precisam passar a mesma mensagem [...]." (Prof. D)

“Eu acho que é questão do relacionamento interdisciplinar, multiprofissional, importantíssima a questão da comunicação [...]." (Prof. F) 
Assim, percebe-se que as limitações em relação ao trabalho em equipe estão mais relacionadas aos mecanismos para efetivar essa interação, do que à crença na importância de tais ações. Um dos fatores que pode estar interferindo na integração entre os profissionais é a atuação transitória de um grande número de estudantes de graduação e pós-graduação que desenvolvem atividades profissionalizantes no cenário do estudo, tendo em vista que a instituição configura-se como um hospital universitário.

A alta rotatividade de profissionais foi apontada por Colomé e Lima (2006) como um dos principais desafios para a efetivação do trabalho em equipe, pois as idas e vindas de profissionais dificulta a integração e construção de vínculo entre eles, assim como a consecução de objetivos comuns capazes de solucionar os problemas de saúde de forma integral.

A operacionalização da integralidade na organização da assistência esbarra nos limites da transposição do conceito para novas práticas, o que limita a integralidade à justaposição de ações de caráter preventivo ou coletivo com as de cunho curativo individual, sem o rearranjo terapêutico e tecnológico necessário à efetivação concreta da integralidade na produção do cuidado (Pinheiro, Mattos, 2006).

Para superação dessa dicotomia, é necessário repensar a formação dos profissionais de saúde. Os entrevistados, ao relacionarem situações práticas à conceituação de integralidade, corroboraram a necessidade de a formação do profissional de saúde contemplar os princípios que regem o sistema de saúde vigente no país, o trabalho em equipe e a atenção integral à saúde. Quanto mais ampla for sua compreensão acerca da organização da rede de atenção, e mais dimensões da integralidade os profissionais incorporarem na sua base teórica, melhor elas serão refletidas nas suas práticas cotidianas (Ceccim, Feuerwerker, 2005).

Apenas dois profissionais apontaram a integralidade segundo dimensões múltiplas, ou seja, abordando a organização do serviço e sua articulação na rede de assistência:

\footnotetext{
“Há várias coisas: ver a saúde como um todo, dar condições de saúde, moradia, resgate da saúde, em um patamar mais abrangente que a doença, dar condições para alcançar o bem estar. Abrange o sistema de saúde. Todos os profissionais vendo o ser humano como um todo." (Prof. A)

“Integralidade eu acho que é uma integração entre a saúde, a saúde da comunidade que seria os postos de saúde, os hospitais, teria que ter um conjunto bom, mas na verdade não temos uma assistência de saúde comunitária boa para essas crianças, eu acho que não tem muito." (Prof. B)
}

Apesar disso, conforme Cecílio (1997, p.301), esse aspecto é de fundamental importância, tendo em vista que os hospitais estão inseridos em uma ampla rede de assistência, atuando como "um local para promoção de saúde e defesa da vida e da cidadania, com suas equipes colaborando ativamente na construção de novas relações dentro do sistema de saúde".

A terceira dimensão da integralidade, caracterizada pelas políticas públicas de saúde e iniciativas governamentais com vistas à integralidade, foi identificada em apenas uma das falas dos profissionais, o que remete, mais uma vez, ao enfoque nas ações cotidianas.

Todavia, os profissionais mencionaram a relevância e necessidade de ações ao nível governamental para viabilização de práticas de integralidade, presente nos elementos elencados como indispensáveis à atuação profissional pelos respondentes:

"[...] são necessárias melhores condições de infra-estrutura, maior quantitativo de pessoal, isso faz com que tenhamos um serviço mais qualificado." (Prof. F)

Nesse sentido, a dimensão das políticas governamentais está relacionada à organização do sistema de saúde, com destaque para novas proposições e desenvolvimento de práticas de gestão democráticas e descentralizadas, a partir da participação dos sistemas locais de saúde e inserção da população na elaboração de políticas e práticas em saúde (Pinheiro, Ferla, Silva Junior, 2007). 
Por esse meandro, observa-se que, conforme Ayres (2001), os serviços de saúde configuram-se como espaços públicos onde se encontram sujeitos coletivos, os quais são produtos de suas subjetividades, no entanto ainda carentes de um agir político, socializado, ciente e comprometido com o seu papel social no contexto das práticas de saúde.

Dessa forma, os profissionais entrevistados associam a integralidade mais facilmente à dimensão relativa à sua atuação, buscando desenvolver práticas que englobem não só a assistência, mas também a prevenção e promoção de saúde de acordo com o contexto socioeconômico da criança atendida no âmbito hospitalar. Pontua-se que, para a materialização da integralidade no cotidiano dos serviços de saúde, é necessário maior diálogo entre os atores que participam da produção do cuidado, buscando o aprimoramento das práticas de gestão e de organização do trabalho em saúde.

\section{Considerações finais}

Constatou-se que a integralidade está presente na base conceitual e nas finalidades que norteiam as práticas dos profissionais da equipe pesquisada, a partir da busca pela articulação de ações destinadas tanto à assistência como à prevenção de agravos e/ou complicações decorrentes do processo patológico que ocasionou a internação.

Entretanto, identificou-se que os profissionais buscam isoladamente uma abordagem integral à criança hospitalizada, o que limita suas ações ao seu campo de atuação, não efetivando um trabalho de discussão, planejamento e execução de ações de forma articulada. A interação no trabalho em equipe só foi evidenciada no atendimento a casos de emergência, quando os profissionais atuam de forma integrada em prol de uma assistência eficaz e resolutiva, mas de forma restrita à dimensão técnica.

Com relação aos três conjuntos de sentidos da integralidade, as ações relatadas pelos profissionais estão centradas na dimensão relativa à atuação profissional. No entanto, para a construção e efetivação das práticas de integralidade nos serviços de saúde, independente do nível de atenção, é necessário que os profissionais, além de pautar suas condutas na busca de uma atenção integral, desenvolvam sua competência política de forma responsável e comprometida com a reorganização dos processos de trabalho, buscando a superação do modelo hegemônico de atenção à saúde.

Especificamente no contexto do trabalho em uma unidade de internação pediátrica, é necessário que os profissionais, ao desenvolverem práticas de cuidado destinadas à integralidade, estejam mais atentos aos anseios e manifestações da criança enquanto indivíduo que deseja ser ouvido e é capaz de expressar sua subjetividade. A hospitalização pode ser um momento ímpar no desenvolvimento emocional e cognitivo da criança, a partir da realização de atividades de educação em saúde, de acordo com sua maturidade compreensiva, buscando situá-la no contexto da ocorrência de doença que está vivenciando e das estratégias de autocuidado que pode desenvolver com a ajuda e/ou supervisão dos seus familiares cuidadores.

Assim, com esta pesquisa, pretendeu-se ampliar a discussão acerca dos caminhos para efetivação do SUS, por meio da análise e discussão das práticas de integralidade desenvolvidas por uma equipe de profissionais da saúde de uma unidade de internação pediátrica. Os resultados encontrados, mesmo relacionados especificamente a uma unidade de internação pediátrica, potencializam importantes reflexões com relação ao trabalho em saúde, ao trabalho em equipe e à integralidade nos serviços de saúde, podendo conduzir a um repensar das práticas e posturas profissionais.

Aponta-se a necessidade de que outras investigações sejam realizadas acerca das diretrizes do SUS e as práticas desenvolvidas no contexto hospitalar, uma vez que os seus princípios devem orientar a produção do cuidado em todos os níveis de atenção à saúde. 


\section{Colaboradores}

Os autores Débora Luiza dos Santos e José Luís Guedes dos Santos realizaram a coleta dos dados, análise e discussão dos resultados, escrita e formatação do texto. Maria de Lourdes Rodrigues Pedroso participou da elaboração e revisão do artigo. Adelina Giacomelli Prochnow orientou todas as etapas do estudo, e Maria Alice Dias da Silva Lima participou da revisão e aprovação final do manuscrito.

\section{Referências}

AYRES, J.R. Sujeito, intersubjetividade e práticas de saúde. Cienc. Saude Colet., v.6, n.1, p.63-72, 2001.

BRASIL. Resolução número 196, de 10 de outubro de 1996. Dispõe sobre as diretrizes e as normas regulamentadoras de pesquisa envolvendo seres humanos. Brasília: Conselho Nacional de Saúde, 1996.

CECCIM, R.B.; FEUERWERKER, L.M. Mudança na graduação das profissões de saúde sob o eixo da integralidade. Cad. Saude Publica, v.20, n.5, p.1400-10, 2004.

CECILIO, L.C.O. Modelos tecnoassistenciais: da pirâmide ao círculo, uma possibilidade a ser explorada. Cad. Saude Publica, v.13, n.3, p.469-78, 1997.

COLOMÉ, I.C.S.; LIMA, M.A.D.S. Desafios do trabalho em equipe para enfermeiras que atuam no Programa Saúde da Família (PSF). Rev. Gaucha Enferm., v.27, n.4, p.548-56, 2006.

FRANCO, T.B.; MERHY, E.E. Programa de Saúde da Família (PSF): contradições de um programa destinado à mudança do modelo tecnoassistencial, In: MERHY, E.E. et al. (Orgs.). O trabalho em saúde: olhando e experenciando o SUS no cotidiano. São Paulo: Hucitec, 2004. p.37-54.

MARQUES, G.Q.; LIMA, M.A.D.S. Organização tecnológica do trabalho em um pronto atendimento e a autonomia do trabalhador de enfermagem. Rev. Esc. Enferm. USP, v.42, n.1, p.41-7, 2008.

MARTINS, M.I.M. A transição tecnológica na saúde: desafios para a gestão do trabalho. Trab., Educ. Saude, v.2, n.2, p.287-310, 2004.

MATTOS, R.A. A integralidade na prática (ou sobre a prática da integralidade). Cad. Saude Publica, v.20, n.5, p.1411-6, 2004.

Os sentidos da integralidade: algumas reflexões acerca de valores que merecem ser defendidos. In: PINHEIRO, R.; MATTOS, R.A. (Orgs.). Os sentidos da integralidade. Rio de Janeiro: UERJ/IMS/Abrasco, 2001. p.39-64.

MENOSSI, M.J.; LIMA, R.A.G.; CORREA, A.K. A dor e o desafio da interdisciplinaridade no cuidado à criança. Rev. Latino-am. Enferm., v.16, n.3, p.489-94, 2008.

MERHY, E.E. Em busca do tempo perdido: a micropolítica do trabalho vivo em saúde. In: MERHY, E.E.; ONOKO, R. (Orgs.). Agir em saúde: um desafio para o público. São Paulo: Hucitec, 1997. p.71-112.

MINAYO, M.C.S. O desafio do conhecimento: pesquisa qualitativa em saúde. 10.ed. Rio de Janeiro: Abrasco, 2007.

PEDUZZI, M. Equipe multiprofissional de saúde: conceito e tipologia. Rev. Saude Publica, v.35, n.1, p.103-9, 2001.

PEROSA, G.B.; GABARRA, L.M. Explicações de crianças internadas sobre a causa das doenças: implicações para a comunicação profissional de saúde-paciente. Interface Comunic., Saude, Educ., v.8, n.14, p.135-48, 2004. 
PEROSA, G.B.; RANZANI, P.M. Capacitação do médico para comunicar más notícias à criança. Rev. Bras. Educ. Med., v.32, n.4, p.468-73, 2008.

PINHEIRO, R.; MATTOS, R.A. Implicações da integralidade na gestão da saúde. In: PINHEIRO, R.; MATTOS, R.A. (Orgs.). Gestão em redes: práticas de avaliação, formação e participação na saúde. Rio de Janeiro: UERJ/IMS/Abrasco, 2006. p.11-26.

PINHEIRO, R.; FERLA, A.; SILVA JÚNIOR, A.G. Integrality in the population's health care programs. Cienc. Saude Coletiva, v.12, n.2, p.343-49, 2007.

PINHO, I.C.; SIQUEIRA, J.C.B.A.; PINHO, L.M.O. As percepções do enfermeiro acerca da integralidade da assistência. Rev. Eletron. Enferm., v.8, n.1, p.42-51, 2006. Disponível em: <http://www.revistas.ufg.br/index.php/fen/article/view/945/1156>. Acesso em: 26 jul. 2008.

QUEIROZ, M.V.; JORGE, M.S. Estratégias de educação em saúde e a qualidade do cuidar e ensinar em pediatria: a interação, o vínculo e a confiança no discurso dos profissionais. Interface - Comunic., Saude, Educ., v.10, n.19, p.117-30, 2006.

RIBEIRO, C.A.; ANGELO, M. O significado da hospitalização para a criança pré-escolar: um modelo teórico. Rev. Esc. Enferm. USP, v.39, n.4, p.391-400, 2005.

SOARES, V.V.; VIEIRA, L.J.E.S. Percepção de crianças hospitalizadas sobre realização de exames. Rev. Esc. Enferm. USP, v.38, n.3, p.298-306, 2004.

TURATO, E.R. Tratado da metodologia da pesquisa clínico-qualitativa: construção teórico-epistemológica, discussão comparada e aplicação nas áreas da saúde e humanas. 2.ed. Petrópolis: Vozes, 2003.

SANTOS, D.L. et al. La integración en las acciones del equipo de salud de una unidad de internación pediátrica. Interface - Comunic., Saude, Educ., v.13, n.31, p.359-68, out./dez. 2009.

La noción de integración engloba tres conjuntos de sentidos: la práctica de los profesionales de salud, los atributos de la organización del servicio y las respuestas gubernamentales a los problemas de salud. Este estudio ha tenido como objetivo analizar y discutir los sentidos relacionados a la integración en las acciones del equipo de salud de una unidad de internación pediátrica de un hospital universitario en el interior del estado de Rio Grande do Sul, Brasil. Se trata de una investigación cualitativa cuyos datos se recogieron a partir de entrevistas y analizados mediante análisis temático. La integración en la práctica de los profesionales se centra en la dimensión relativa a la actuación profesional en la que tratan de asociar asistencia y prevención, vislumbrando al niño hospitalizado en su contexto económico y social. Señalan la importancia del trabajo de equipo pero encuentran dificultades al efectivarlo, lo que perjudica la articulación de las acciones en pro de una atención integral.

Palabras clave: Asistencia integral a la salud. Equipo de asistencia al paciente. Práctica profesional. Servicios del hospital. Pediatría. 